\title{
PREVENCIÓN DEL MALTRATO ENTRE IGUALES A TRAVÉS DE LA EDUCACIÓN MORAL
}

\author{
PREVENTION OF BULLYING ACROSS THE MORAL EDUCATION \\ José AVILÉs M. ${ }^{1}$ \\ Universidad de Valladolid, Departamento de Orientación del IES Parquesol de Valladolid (España) \\ (RECIBIDO 11/01/2012 - ACEPTADO 30/05/2012)
}

\begin{abstract}
RESUMEN
Se analizan las acciones llevadas a cabo en España para abordar el bullying (maltrato entre iguales) desde diferentes ámbitos competenciales en aspectos de normativización, formación, divulgación, sensibilización, prevención e intervención. Igualmente se ponen de relieve las líneas de actuación preferentes en prevención e intervención en la comunidad educativa incidiendo en el compromiso y la coordinación entre el profesorado y las familias haciendo protagonista al alumnado como parte de la solución en la erradicación del bullying.

El texto reflexiona sobre las claves de la educación moral en relación con los componentes que identifican el maltrato entre iguales (bullying), su abordaje escolar y las estrategias educativas necesarias para llevar a cabo el trabajo en los planos cognitivo, emocional y conductual con el alumnado por parte del profesorado y en colaboración y coordinación con las familias.
\end{abstract}

Palabras clave: Bullying, ciberbullying, víctimas, agresores/as, educación moral, prevención del maltrato.

\begin{abstract}
The actions carried out in Spain are analyzed to approach the bullying from different areas in aspects of legislation, formation, spreading, awareness, prevention and intervention. Equally the preferential lines of action are emphasized in prevention and intervention in the school community affecting in the commitment and the coordination between the teachers and the families, making the students participate and cooperate in the solution as well as the facing of the bullying.

The text examines about the keys of the moral education in relation with the components that identify the bullying, its coping with the skills and the necessary educational strategies to carry out the work in the cognitive, emotionally and behaviourally phases with the students on the part of the teachers and in collaboration and coordination with the families.
\end{abstract}

Keywords: Bullying, cyber bullying, victims, aggressors, moral education, preventive strategies of bullying

1 Profesor del Departamento de Psicología de la Universidad de Valladolid.

Área de Personalidad, Evaluación y Tratamiento Psicológico.. Jefe del Departamento de Orientación del IES Parquesol de Valladolid, España. E-mail: jmaviles@psi.uva.es 


\section{INTRODUCCIÓN}

El maltrato entre iguales (bullying) es hoy un problema en muchos contextos escolares que está aún pendiente de abordarse efectivamente en el seno de las comunidades educativas. Este fenómeno ha sido puesto de relevancia en numerosos estudios de investigación (Avilés, 2006a, Olweus, 1983, 1998, Ortega, 1992; Smith, 1999) en contextos educativos e institucionales (Defensor del Pueblo, 1999, 2007). Estos estudios han mostrado las aristas del bullying con sus peculiaridades intrínsecas (naturaleza y tipología del maltrato), de evolución histórico-social (ciberbullying) así como de orden contextual, en sus manifestaciones locales, regionales o nacionales. La mayoría de ellos han sido estimulados por la necesidad política, social y educativa de acotar y abordar el fenómeno. Por poner el ejemplo de algunos de los llevados a cabo en España, además de los señalados anteriormente, han sido relevantes otros que han abarcado el fenómeno en un marco nacional (Centro Reina Sofía, 2005), autonómico (Consejo Escolar de Andalucía, 2006; Ararteko, 2006; Díaz-Aguado, Arias y Martín, 2004, 2005 y 2006; ECESC, 2006; Gobern de les Illes Balears 2005; Gobierno Vasco, 2004; Gómez Bahíllo, Puyal, Elboj, Sanz, y Sanagustín, 2005; Oñederra, Martínez, Tambo y Ubieta, 2005a,b; Sáenz, Calvo, Fernández y Silván, 2005; Sindic de Greuges, 2006) o local (Avilés y Monjas, 2005; Ramírez, 2006 ).

Al mismo tiempo y por la importancia psicológica, social y educativa de estas situaciones ha habido iniciativas de diversa índole para atacar el problema con diversos grados de acierto (Zaitegi, Fernández, Uruñuela, Avilés, Boqué y Gómez, 2010). Así, se han puesto en marcha actuaciones que han ido desde lo normativo a lo formativo pasando por las campañas de sensibilización social y profesional y la elaboración de propuestas de prevención e intervención. Sin embargo, aunque en el presente artículo nombraremos aquellas actuaciones más relevantes y meritorias para abordar el maltrato entre iguales desde el punto de vista preventivo y de intervención, el objetivo de nuestro trabajo va a ir encaminado a poner el punto de mira en lo que creemos que es el núcleo gordiano a través del que deben girar las acciones que se deben llevar a cabo en el seno de las comunidades educativas que gestionan y tienen posibilidades de erradicar esa lacra, y que no es otro que el tratamiento preventivo y profesional de los contravalores que fundamentan y vehiculan el maltrato entre iguales, y que tienen que ver con el grado de criterio moral de que dispone el alumnado, su educación moral (Avilés, 2012 en prensa; Avilés, Irurtia, G ${ }^{\mathrm{a}}$-López y Caballo, 2011). Esa es nuestra propuesta. Sobre cómo organizar y llevar a cabo de forma intencional el trabajo, el entrenamiento y el aprendizaje de los contravalores que contrarrestan los efectos del bullying y el ciberbullying en la escuela, a través de espacios y tiempos planificados por los profesionales educativos, y en colaboración y coordinación con los sectores de la comunidad educativa implicados. Todo ello, vertebrado a través de instrumentos útiles que estén legitimados por sus usuarios y beneficiarios, como son los Proyectos Antibullying (Avilés, 2005). 


\section{INICIATIVAS PARA ABORDAR EL MALTRATO ENTRE IGUALES}

Sin duda han sido muchas las actuaciones para abordar un fenómeno tan relevante desde el punto de vista psicológico y educativo. Incluso, todas ellas participan de una secuencia o un patrón de ocurrencia con puntos de coincidencia. Por razones obvias, en el relato nos centraremos en el contexto socioeducativo español a la hora de ilustrar estos procedimientos, aunque, como decimos, haremos referencia a procesos similares en otros países.

- Desencadenantes sociales: en muchos países el 'proceso histórico' ha sido muy similar, se han producido incidentes críticos en lo social y en lo educativo que han provocado el debate social abierto sobre la necesidad de atajar el sufrimiento que muchos alumnos/as y muchas familias estaban padeciendo por las consecuencias del maltrato entre iguales.

El caso Jokin en España (en septiembre de 2004 un adolescente guipuzcoano -Jokin- muere tras lanzarse desde la muralla de su pueblo, Fuenterrabía, al no poder soportar el acoso al que estaba siendo sometido durante tiempo por parte de un grupo de compañeros) hizo que nada fuera igual desde entonces en nuestro país sobre el bullying. Desencadenantes similares se produjeron en otros países (Fante, 2004; Olweus, 1983, Rigby y Slee, 1999; Salmivalli, Karhunen y Lagerspetz, 1996; Smith, 1999; Sullivan, 2001; Ziegler y Rosenstein-Manner, 1991) a partir de los que se construyeron iniciativas de diversa índole.

- Reformas legislativas: las reformas legislativas que se han puesto en marcha en el contexto español se han establecido en el marco de competencias de las respetivas administraciones, a nivel nacional y autonómico, especialmente en este último caso en lo que se refiere a la promulgación de leyes y desarrollos normativos y reglamentarios (Gómez Rivas, 2011). Fundamentalmente el trabajo normativo se ha centrado en la ordenación de instrumentos reguladores de la convivencia escolar y, dentro de ella, el tratamiento concedido al bullying. Entre las iniciativas más significativas destacamos:

Los observatorios para la convivencia escolar. A nivel estatal o regional desde el año 2004 a 2007, se han creado distintos "Observatorios de Convivencia" en la mayoría de Comunidades Autónomas, asignándoles funciones de coordinación, consulta, análisis y evaluación del estado de la convivencia escolar en las comunidades educativas.

Se han regulado los premios que anualmente se conceden a nivel estatal y regional a las 'buenas prácticas de convivencia escolar. Se han convocado concursos nacionales de buenas prácticas de convivencia entre 2007 y 2011 difundiendo a través de sus memorias ejemplos sobre cómo trabajar la convivencia en positivo en los centros escolares. Otros premios que tienen relación con la convivencia han sido los premios 'Irene: la paz empieza en casa' que valoran las experiencias, investigaciones y materiales educativos que contribuyen a prevenir y erradicar las conductas violentas, a promover la igualdad real entre mujeres y hombre y a desarrollar estrategias desde el sistema educativo para erradicar la violencia de 
género. Otros premios han sido los 'Miguel Hernández' que reconocen y divulgan las acciones inclusión educativa, los premios "Marta Mata" a la calidad y mejora de la acción educativa, y los premios de "Educación y Seguridad en el Entorno Escolar", relacionados con la seguridad ciudadana y la protección civil.

Se ha normativizado la concesión de subvenciones también para realizar proyectos o actividades de fomento de la educación y la cultura de paz para asociaciones -no para centros escolares- entre los años 2.006 y 2008, con la intención implicar a más agentes educativos en el trabajo de la convivencia escolar.

Ha habido una normativa de ordenación de los derechos y deberes del alumnado -todas las administraciones regionales han elaborado normativa para regular estos derechos y deberes, en varios casos, de todos los componentes de la comunidad educativa-, los planes de convivencia en los centros y la legislación sobre programas y leyes regionales. Se ha pretendido ofrecer a la comunidad educativa indicaciones de planificación, organización y funcionamiento relativas a la convivencia escolar apostando por medidas diversas como, entre otras, la consideración de autoridad pública del profesor/a, con los efectos que le reconoce el ordenamiento jurídico; el apoyo a la mediación escolar como medio para la resolución de los conflictos; la creación de 'aulas de convivencia' para la gestión de la indisciplina o la disrupción; la elaboración de protocolos de actuación frente al maltrato entre iguales; la atribución de competencias disciplinarias a los órganos de gobierno de los centros escolares; el establecimiento de mecanismos de conciliación entre familias y centro educativo; ...etc. Sin embargo, ha habido notables diferencias de orientación en la aplicación de estas medidas en función de prisma educativo y político de quienes toman esas decisiones normativas. Así, en unos casos se habla de corrección frente a términos como sanción; se incluyen o no respuestas y de qué tipo al fenómeno del bullying, su prevención y tratamiento; se da más o menos trascendencia a la mediación; se crean o no y se regulan o no las aulas de convivencia; se elevan o rebajan las competencias disciplinarias y de fomento de la convivencia atribuidas al equipo directivo, tutores, profesorado, Comisiones de Convivencia o Consejo Escolar; se establecen compromisos de conciliación o no entre las familias y el centro escolar con objeto de establecer mecanismos de coordinación y colaborar en la aplicación de medidas tanto en el tiempo escolar como en el tiempo extraescolar; ...etc..

En algunos casos, se han regulado los protocolos de actuación frente al bullying facilitando indicaciones para el tratamiento de los casos en cuanto al papel y actuaciones que tienen que realizar los diversos agentes educativos (Departamento de Educación, 2011). Sin embargo, con carácter general, normativamente hablando se ha legislado no específicamente sobre el bullying, sino más bien sobre la convivencia en general donde se insertan los procesos de violencia entre escolares. Aunque en algunos países, como en Perú, ha habido normas que se han dirigido específicamente a atajar este problema con rango de ley general y con desarrollos reglamentarios (Ley 29719). 


\section{MEDIDAS DE PREVENCIÓN Y DE INTERVENCIÓN}

Campañas informativas sobre acoso escolar: Dirigidas a la población en general y en particular a la comunidad escolar, suelen difundir sus mensajes en folletos, pegatinas, carteles, pines y trípticos divulgativos para llegar a la sociedad y los diferentes sectores de la comunidad educativa; algunas informaciones también toman cuerpo en cuñas de radio, anuncios en televisión para concienciar a la sociedad en general.

Programas de concienciación y sensibilización: el objetivo de estos programas (Fernández y Hernández, 2005; Grasa, Lafuente, López y Royo, 2006; Monjas y Avilés, 2005) ha sido la puesta en valor de la importancia que la erradicación del bullying tiene para el bienestar socioemocional del alumnado y su rendimiento académico en sectores que, tradicionalmente, no le han dado esa importancia. Pretenden erradicar la trivialización del fenómeno en la sociedad, en las familias, entre el alumnado y movilizar al profesorado para que dé un paso adelante en su tratamiento y combate en el aula.

Elaboración de guías de actuación para los profesionales educativos: las administraciones con responsabilidades educativas han puesto en marcha mecanismos de edición de pautas educativas para proveer a los profesionales de la educación de herramientas de trabajo para el tratamiento del maltrato entre iguales. En los últimos cinco años se han desarrollado diversos instrumentos en distintas comunidades autónomas en España (Asturias, 2007; Canarias, 2009; Baleares, 2005 Extremadura, 2008; Madrid, 2006; País Vasco, 2005, 2007).

Realización de foros formativos para la prevención y la intervención del bullying: desde distintos ámbitos, profesionales, nacionales, regionales y locales, se han promovido acciones formativas de capacitación de profesionales y no profesionales que de alguna manera están en relación con el fenómeno del maltrato entre iguales. Universidades (formación inicial y continua), Administraciones Públicas, Centros de Formación del Profesorado, Expertos e Investigadores, etc. han puesto en manos de los profesionales de la educación las herramientas de prevención y de intervención en el tratamiento del bullying a través ce Congresos, Simposios, Cursos, Seminarios, Grupos de Investigación, Formación on line, etc. En este último aspecto, destacamos iniciativas como el VISTOP 2008 y el VISTA 2008. Por otra parte, es básico recalcar la idoneidad formativa para el bullying de una modalidad muy útil para su abordaje, la formación de la comunidad educativa en el propio centro, contexto ecológico en el que es más efectiva la intervención.

Orientaciones profesionales a los distintos sectores de la Comunidad Educativa (Avilés, 2006b; Collell y Escudé, 2004; Díaz-Aguado, 2006). Con un carácter más especializado y con la intención de aportar herramientas profesionales de lucha contra el bullying también aparecen orientaciones específicas para el profesorado, las familias, etc. que aportan instrumentos más técnicos y prácticos de lucha contra el acoso donde se produce.

Intervención de expertos y de equipos de actuación urgente: en algunos casos la participación de expertos ha sobrepasado el campo de la colaboración teóricopractica en la intervención sobre el acoso y se establecido a través de comités estables y formalizados, foros consultivos, observatorios, consejos orientadores, 
etc. En otras ocasiones se ha recurrido al perfil profesionalizante del experto para pedirle actuaciones de tipo psicológico, reeducador o sancionador. En este sentido han funcionado, por ejemplo en Canarias o Valencia, equipos mixtos de inspectores, psicólogos o educadores que con carácter inmediato han actuado en diferentes lugares en que se han producido casos de acoso o denuncias de los mismos.

Establecimiento de sistemas de comunicación rápida y segura: con el fin de facilitar a las víctimas del acoso y sus familias mecanismos rápidos y seguros de comunicación se han establecido iniciativas prácticas como teléfonos anónimos, correos electrónicos, páginas web o sistemas de teleayuda (Avilés, Torres y Vián, 2008; Cowie y Fernández, 2006), que a través de correos electrónicos gestionados por mediadores adultos evitan la identificación de los comunicantes, permitiendo la comunicación y orientación entre iguales en casos de bullying.

Actuaciones profesionales en los contextos educativos: sin duda es la parte fundamental para hacer que el bullying desaparezca o se minimice en el medio educativo. Aquí digamos que la puesta en práctica de medidas tiene un recorrido más largo en el tiempo y de construcción más elaborada y compleja, pues exige la cooperación, coordinación y actuación de los agentes educativos que tienen responsabilidad en ese contexto. No obstante, existen actuaciones que se han mostrado positivas y con efectividad en el tratamiento y también sabemos que hay premisas básicas para asegurar su eficacia como se pone de manifiesto en muchas investigaciones (Smith, Pepler y Rigby, 2004). Entre ellas queremos señalar, el mantenimiento en el tiempo de las iniciativas institucionales y educativas, la implicación del profesorado en el trabajo de prevención e intervención del acoso, la construcción de estructuras (organizativas, formativas, ...) que actúen activamente contra el bullying en la escuela, el protagonismo del alumnado en las iniciativas y salidas al problema del maltrato o el trabajo coordinado en una cultura antibullying en la comunidad educativa.

Entre las actuaciones efectivas que ayudan a la erradicación del bullying entre escolares indicaremos algunas que comprometen a distintos sectores educativos, profesorado, familias, equipos directivos, alumnado, administraciones educativas, etc. (Avilés, Irurtia, Ga ${ }^{\mathrm{a}}$-López y Caballo, 2011). Sin duda, cada una de ellas podría tener, quizás en otro espacio, un desarrollo mucho más exhaustivo que estas meras indicaciones:

1. Existencia de un Proyecto Antibullying (Avilés, 2005) en el seno de la comunidad educativa.

2. Decisión política institucional y educativa efectiva de los órganos de gobierno del centro para actuar contra el acoso.

3. Una estructura organizativa (Grupo de Trabajo Antibullying) que con carácter estable gestiona situaciones relacionadas con el bullying.

4. Respuestas preparadas (protocolos) de actuación para cuando se tienen sospechas, confirmación y constancia de situaciones de bullying en todas sus modalidades, incluido el ciber bullying. 
5. Inserción curricular del trabajo contra el maltrato entre iguales en la agenda docente del profesorado como tema transversal de abordaje intencional entre los de la mejora de la convivencia. Tratamiento de la educación emocional y de la afectividad (Avilés y Alonso, 2012) así como del potencial solidario y de servicio con el alumnado (Uruñela y Avilés, 2012).

6. Creación de redes o estructuras estables de prevención de los conflictos en la convivencia en la comunidad educativa (Avilés, Alonso y Mascarenhas, 2012) en la que se integren de forma equilibrada sus miembros, equipos de mediación (Boqué, 2005), círculos de convivencia entre profesorado y familias (Olweus, 1998), equipos de ayuda (Avilés, Torres y Vián, 2008), acuerdos reeducativos (Alonso y Avilés, 2012), tutores de convivencia (Avilés, García y Mateu, 2012), etc.

7. Existencia de una intervención compartida, discutida y consensuada en los círculos mixtos de participación y formación entre profesorado, alumnado y familias.

8. Orientación sistémica y social de la intervención, preferentemente al grupo de convivencia (a las dinámicas internas) y al contexto en que se produce el bullying o el ciber bullying y no solo hacia los individuos implicados, sean víctimas y/o agresores/as.

9. Abordaje de los contenidos de educación moral como referente formativo y de intervención en las iniciativas de intervención contra el maltrato. Trabajo colectivo en las tareas tutoriales del profesorado con el alumnado y entre los objetivos de formación integral compartida entre familias y profesorado para con el alumnado.

Elaboración de materiales de trabajo en el aula: el trabajo de prevención e intervención cotidiano en los centros educativos ha ido generando algunos materiales y estrategias de trabajo que merece la pena señalar como idóneas para su abordaje, aunque sea sucintamente por lo limitado de espacio que disponemos. Muchas de ellas tienen un corte audiovisual, a través del visionado de videos que cuentan historias o expresan facetas del problema que pueden ser tratadas y opinadas por el alumnado. En formato más largo, también nos hemos servido de películas que abordan el problema directamente, es el caso en castellano de "Bienvenidos a la casa de muñecas" 'Cobardes' o "Amigas hasta la muerte". También ha servido el teatro expresamente construido para ser visto y tratar el tema o el ejercicio de la dramatización con el alumnado para el juego de roles que permite a los protagonistas de la representación tomar perspectivas desde los distintos papeles implicados en el problema, siendo guiados y orientados por el tutor o monitor con intervenciones en aquellos aspectos que interesan. Otro recurso frecuentemente utilizado es el de los juegos didácticos, por ejemplo a través del manejo de los derechos humanos como contenido del juego. Cuentos y cómics han sido utilizados para, a través del contenido narrativo, explorar situaciones de riesgo o reflejar vivencias emocionales de algunos de los implicados. Estas técnicas narrativas o autonarrativas han servido para la expresión y gestión de los sentimientos y las emociones, especialmente en niños de menos edad donde existe mayor dificultad para manejar y controlar estos procesos. Sin embargo, las situaciones más ricas 
y creativas se han producido en las situaciones directas ocurridas en los centros educativos, a través de las soluciones que estos han buscado para dar respuesta de forma imaginativa e innovadora a las situaciones, en el cotidiano del aula, en la gestión de los conflictos a través de la resolución colaborativa de los mismos, a través del análisis de casos, del uso de los relatos, la poesía, el trabajo de los valores y de los contenidos de la educación moral, en el juicio reflexivo y colectivo de las situaciones que vivimos cada día en las relaciones interpersonales en el grupo de convivencia.

\section{EL RETO DE LA EDUCACIÓN MORAL}

Sin duda, en el plano interno de los procesos que mantienen vivo el bullying y/o el ciberbullying existen contravalores que cuestionan el más mínimo criterio moral, y que es a través de su transformación, como lograremos que el alumnado aprenda a reflexionar sobre los hechos, gestione adecuadamente sus sentimientos y emociones y autorregule interactivamente las conductas de abuso e intimidación.

Las estrategias y las tareas de intervención han de abarcar los planos cognitivo, afectivo y conductual si queremos que su recorrido sea completo, por lo que hemos de asegurar su desarrollo en los tres planos.

Para ello, nos ayudará tener presentes los componentes (Avilés y Alonso, 2008; Avilés, 2012 en prensa) del maltrato entre iguales. Es con estas constantes con las que venimos trabajando tanto en la prevención del maltrato presencial como en el virtual. Cuando hemos analizado estos componentes lo hemos hecho en cuatro campos de análisis: el interpersonal, el intrapersonal, el intergrupal y el contextual. En lo interpersonal destacamos componentes de desequilibrio de poder fundamentalmente entre víctima y agresor/a. Además, en el agresor/a encontramos componentes de cálculo de riesgo/beneficio (Avilés, 2010), control y dominancia en función de la modalidad de bullying presencial o ciberbullying, mientras que en la víctima es palpable su sumisión la inseguridad y la vulnerabilidad interpersonal. Por todo ello, hablamos de componentes de control, seguridad y poder.

Si analizados el plano intrapersonal los procesos internos de víctima y agresor/a son diferentes. Mientras que en la víctima hemos de manejar la indefensión aprendida, la imprevisibilidad y la confusión, en el agresor/a nos enfrentamos a la falta de empatía y cierta distorsión emocional y cognitiva. En el caso del ciberbullying añadimos en el agresor/a los déficits en pensamiento consecuencial y falta de claves socioemocionales (Mason, 2008) al ejecutar sus ataques sin la presencia de la víctima.

Desde el punto de vista intragrupal se ponen en juego las reglas de regulación social, el efecto desinhibidor de la agresión que se hace en grupo y los aspectos de identidad grupal, donde en el individuo se priman aspectos de afiliación y de pertenencia. 
Por último, en el plano contextual, es necesario manejar los conceptos de 'exposición pública' de la agresión y el conocimiento de la misma por parte de los demás, que son distintos si hablamos de bullying o de ciberbullying, donde la audiencia se agranda y se denomina 'extendida'. Este conocimiento está directamente relacionado con la magnitud del daño. También en el plano contextual y situacional juega un papel decisivo la duración del maltrato, que está en relación directa con el tiempo de sufrimiento de la víctima.

En este sentido, el trabajo educativo tanto en la familia como en el contexto escolar debe dirigirse a abordar los contenidos morales involucrados en esos procesos en los tres planos, el cognitivo, el emocional y el conductual. Señalaremos a continuación algunos contenidos morales. En relación a los componentes subyacentes al maltrato en el plano interpersonal de las relaciones de agresor/a y víctimas, identificamos los siguientes:

1. El des-equilibrio de poder y su ejercicio abusivo debe contrarrestarse con un abordaje de la igualdad, la interiorización de la aceptación y/o la crítica a la autoridad y análisis, aceptación y adopción de patrones de conductas de los indicadores de respeto mutuo.

2. La in-seguridad que mina el estar de la víctima está relacionada directamente en el ejercicio de los derechos, el de seguridad y de bienestar y el derecho a no sufrir daño ni físico ni moral por parte de nadie.

3. El control y descontrol de las situaciones que se encarna en los dos personajes respectivamente vuelve a incidir en los indicadores de respeto al otro y los grados de integridad que deben aprenderse para construir relaciones igualitarias y recíprocas.

El plano intrapersonal de los dos perfiles viene identificado por una falta de conexión emocional en el agresor/a y la indefensión propia y ajena (Avilés, 2006a) en las víctimas. El trabajo se plantea en estas dos bandas:

1. El aprendizaje de la reciprocidad, el altruismo y la generosidad para incidir en los referentes de empatía del agresor/a.

2. El trabajo de autoafirmación y asertividad que fundamenten el trabajo sobre los derechos individuales personales y el sentido de justicia en las víctimas.

El plano grupal supone un trabajo doble en los planos externos e internos al grupo. Desde fuera hemos de trabajar y conocer las redes de poder en el grupo, su cultura grupal, las normas reguladoras de las situaciones de abuso y la construcción de las estructuras como Equipos de Ayuda, Equipos de Mediación, de Mentores, etc. (Avilés, Alonso, y Mascarenhas, 2012) que prevengan que surjan esas conductas en su seno. Internamente al grupo se hace necesario abordar el afrontamiento de la presión grupal, la cooperación en la consecución de tareas grupales y los procesos de independencia emocional en las relaciones vinculares y la toma de decisiones. Todo ello relacionado con valores en sentido proactivo respecto a la ayuda, la compasión, la lástima y/o la piedad. También en este plano interno de 
los procesos grupales será necesario tener presentes y considerar de forma equilibrada los procesos identitarios relacionados con los sentimientos de afiliación y pertenencia al grupo de sus propios miembros.

El trabajo en estos tres planos rebaja necesariamente los efectos que se producen en el plano contextual respecto al daño y el sufrimiento de la víctima, pues deberían suponer la eliminación de los elementos causantes del maltrato.

El trabajo ha de plantearse en todos los ámbitos educativos y de forma coordinada a través de esquemas de trabajo intencionales en los que se ponga al sujeto/ alumno/a en disposición de reflexionar/sentir/aprender/actuar. Este trabajo debe seguir un procedimiento secuenciado de abordaje, especialmente en la escuela a cargo de los tutores de la clase y enmarcado en un programa más amplio de educación en valores integrado en el proyecto curricular y educativo del centro y la comunidad educativa.

Metodológicamente hablando, nos decantamos tanto por sesiones específicas insertas en los períodos lectivos de clase como por intervenciones educativas ad hoc, incluidas en el desarrollo de las distintas materias, como consecuencia del abordaje de unos hechos que pueden suceder en la convivencia cotidiana del grupo. La forma en que mejor interioriza el alumnado estas situaciones es a partir de un trabajo tipo taller, lo cual no quita para que organicemos sesiones más expositivas o de debate.

El objetivo de nuestra intervención ha de encaminarse a conseguir que los individuos actúen conforme a códigos éticos de comportamiento. Estos códigos éticos de conducta hemos de trabajarlos en la escuela, complementariamente al trabajo hecho en la familia en la misma dirección, a través de actuaciones intencionales con el fin de dotar a los individuos de esas herramientas morales. A continuación indicamos algunos de los tópicos más comunes que utilizamos, señalando entre paréntesis los contenidos que se tratan en las sesiones de taller (Avilés, 2012 en prensa; Avilés y Alonso, 2012; Boqué, 2005):

- El respeto al otro y a sus diferencias (reconocimiento, consideración, curiosidad, admiración, capacidad para esperar, mostrar y tener confianza, ajustar las expectativas, evitar los prejuicios, romper los estereotipos...).

- El trabajo de la comunicación (saber decir que no, usar la diplomacia, tener sinceridad, poner en práctica consciente la simpatía, satisfacer la autoafirmación sintiéndose bien...).

- El manejo de los sentimientos y de las emociones (identificarlas en uno mismo y en los otros, compartirlas, afirmarlas, interpretarlas, atribuirlas, poner en práctica la empatía, ser capaz de deducir qué sentimientos y emociones hay en juego, inferirlos...).

El arte de llegar a acuerdos morales aceptados y compartidos (adquirir el hábito de la participación, practicar la renuncia a las propias posiciones y aceptar las propuestas de los otros, comprometerse en los acuerdos y revisarlos cuando pasa un tiempo). 
La promoción de la ayuda (poner en práctica la generosidad, la disposición, el estar pendiente del otro, compartir, saber ayudar en grupo, corresponsabilizarse de los acuerdos, realizarse y sentirse bien ayudando, compadecerse de quien sufre, sentir lástima por quien lo está pasando mal...).

El uso del diálogo para resolver los conflictos (poner en marcha el pensamiento creativo, dedicar tiempo a la observación, no ser cicatero en los acuerdos y sí generoso, sublimar las propias posiciones por un bien superior para ambas partes, sentirse bien al superar situaciones molestas y problemáticas...).

La actuación a partir de un criterio propio (demostrar coherencia entre lo que uno piensa, dice y hace, asumir responsabilidad de sus actuaciones, entrenar el pensamiento consecuencial, poner en práctica el sentido del deber...).

La actuación frente al poder (practicar la obediencia y el respeto frente a la autoridad, oponerse al autoritarismo y al abuso, practicar la asertividad).

La gestión de la presión grupal (saber gestionar la influencia de los líderes, aguantar la presión del grupo, rechazar la dependencia, combatir el sometimiento, aceptar la influencia, no admitir la dominancia...).

El establecimiento de sistemas de justicia restaurativa (reconocimiento de la culpa, restitución del daño, donación del perdón, control grupal de los cambios deseados, ...).

Estos frentes de trabajo son retos hoy en la educación, sin duda. Aunque somos conscientes de que desde algunos ámbitos puedan, incluso, cuestionarse como objetivos educativos, suponen un cuerpo de trabajo imprescindible si lo que queremos abordar es crecimiento moral del alumnado y de nuestros hijos e hijas. Más si lo que queremos es erradicar el maltrato entre iguales a través del ejercicio abusivo del poder.

El profesorado no podrá hacerlo solo, es un trabajo comunitario, incluso diría, social que persigue cambiar las cosas, cambiar el mundo, hacer ciudadanos y ciudadanas más responsables de sus actuaciones y más felices en la convivencia cotidiana con sus iguales.

\section{REFERENCIAS BIBLIOGRÁFICAS}

Alonso, F. y Avilés, J.M. (2012). Los acuerdos reeducativos como instrumento preferente de resolución de las situaciones de indisciplina escolar. En J.L. Pais, I. Leal, A. Pereira, A. Torres, I. Direito y P. Vagos (Eds.), actas del $9^{\circ}$ Congreso de Psicología da Saude, Aveiro: Placebo.

Ararteko (2006) Convivencia y conflicto en los centros escolares. Informe elaborado por E. Martín, J.F. Mujica, K. Santiago, A. Marchesi, E. M. Pérez, A. Martín y N. Álvarez. Vitoria-Gasteiz: Publicaciones del Ararteko.

Avilés, J.M. (2005). Intervenir contra el bullying en la Comunidad Educativa. www. concejoeducativo.org (8 diciembre 2005). 
Avilés, J. M. (2006a). Bullying: el maltrato entre iguales. Agresores, víctimas y testigos en la escuela. Salamanca: Amarú.

Avilés, J. M. (2006b) L'assetjament escolar. Orientacions per a la prevenció i la intervenció. Guia del professorat. Palma de Mallorca: Escola de Mitjans Didactics.

Avilés, J. Ma (2010). Ciberbullying y éxito escolar. En Boletín de Psicología, (98), 73-85.

Avilés, J. Ma (2012 en prensa). Guía de prevención del bullying para el profesorado. Sao Paulo: Mercado de Letras.

Avilés,J.M.-Alonso,M.N. (2008): Análisis de componentes identificativos de la violencia en el contexto escolar.Violencia, conflicto y maltrato. Itinerario de frontera. En I. Leal, J.L. Pais, I. Silva y S. Marques, (Eds.), $7^{\circ}$ Congresso Nacional de psicología da saúde, (pp. 119-129): Porto: ISPA Ediciones.

Avilés, J. M., Torres, N. y Vian, M.V. (2008). Equipos de ayuda, maltrato entre iguales y convivencia escolar. En Revista de Investigación Psicoeducativa. Universidad de Almería.

Avilés, J.M., Irurtia, M.J., G ${ }^{\text {a }-L o ́ p e z, ~ J . ~ y ~ C a b a l l o, ~ V . ~(2011) . ~ B u l l y i n g, ~ e l ~ m a l t r a t o ~ e n t r e ~}$ iguales. En Behavioral Psychology, Vol. 19, N 1, pp. 57-90.

Avilés, J.M., García, N. y Mateu, F. (2012). Tutores/as de convivencia, nuevos perfiles profesionales y educativos para la resolución de los conflictos en la escuela. En J.L. Pais, I. Leal, A. Pereira, A. Torres, I. Direito y P. Vagos (Eds.), actas del $9^{\circ}$ Congreso de Psicología da Saude, pp. 770-777, Aveiro: Placebo.

Avilés, J.M., Alonso, M.N. y Mascarenhas, S. (2012). Los equipos de ayuda como estructura social construida en el aula para la prevención de las situaciones de acoso. En J.L. Pais, I. Leal, A. Pereira, A. Torres, I. Direito y P. Vagos (Eds.), actas del $9^{\circ}$ Congreso de Psicología da Saude, pp. 45-52, Aveiro: Placebo.

Alonso, F. y Avilés, J.M., (2012). Los acuerdos reeducativos como instrumento preferente de resolución de las situaciones de indisciplina escolar. En J.L. Pais, I. Leal, A. Pereira, A. Torres, I. Direito y P. Vagos (Eds.), actas del $9^{\circ}$ Congreso de Psicología da Saude, pp. 400-406, Aveiro: Placebo.

Avilés, J.M. y Alonso, M.N., (2012). La formación de la afectividad y la comunicación del alumnado como base de su protagonismo en la mejora de la convivencia escolar. En J.L. Pais, I. Leal, A. Pereira, A. Torres, I. Direito y P. Vagos (Eds.), actas del $9^{\circ}$ Congreso de Psicología da Saude, pp. 74-82, Aveiro: Placebo.

Boqué, M. C. (2005). Tiempo de Mediación. Barcelona: CEAC.

Centro Reina Sofía para el estudio de la violencia (2005). Informe Violencia entre compañeros en la escuela. Valencia.

Collell , J. y Escudé, C. (2004). Guía per a les families. http://www.xtec.net/ jcollell

Consejo Escolar de Andalucía (2006). Encuesta a representantes de la Comunidad Educativa sobre el estado de la Convivencia en los centros educativos. Consejo Escolar de Andalucía. Consejería de Educación de la Junta de Andalucía. 
Cowie, H. y Fernández, F.J. (2006). Ayuda entre iguales en las escuelas: desarrollo y retos. En Revista Electrónica de Investigación Psicoeducativa, 4 (2), 291-310

Defensor del Pueblo (1999). Informe sobre violencia escolar: El maltrato entre iguales en la Educación Secundaria Obligatoria. Elaborado por C. del Barrio, E. Martín, I. Montero, L. Hierro, I. Fernández, H. Gutiérrez y E. Ochaíta. Madrid: Publicaciones de la Oficina del Defensor del Pueblo. http://www.defensordelpueblo.es/index. asp?destino $=$ informes 2 .asp

Defensor del Pueblo (2007). Violencia Escolar: El Maltrato entre Iguales en la Educación Secundaria Obligatoria 1999-2006. Madrid: Publicaciones de la Oficina del Defensor del Pueblo. http://www.defensordelpueblo.es/ (7 febrero 2007)

Departamento de Educación (2011). Guía de actuación en los centros educativos ante el maltrato entre iguales. Consejería de Educación, Universidades e Investigación. www. hezkuntza.ejgv.euskadi.net/r43.../tratu_txarrak_2011_c.pdf (21 diciembre 2011)

Díaz-Aguado, M. J., Martínez, R., y Martín, G. (2004). Prevención de la violencia y lucha contra la exclusión desde la adolescencia. Madrid: Ministerio de Trabajo y Asuntos Sociales, INJUVE.

Díaz-Aguado, M ${ }^{\mathrm{a}}$. J., (2006). “El acoso escolar y la prevención de la violencia en la familia”. Madrid: Consejería de Familia y Asuntos Sociales.

ECESC (2006), Enquesta de convivència escolar i seguretat a Catalunya. Barcelona: Gencat.

Fante, C. (2004). Fenômeno Bullying. Como prevenir a violência nas escolas e educar para a paz. Campinas: Editora Verus.

Fernández, I. y Hernández, I. (2005). El maltrato entre escolares. Guía para jóvenes. Madrid: Defensor del Menor en la Comunidad de Madrid.

Gómez Bahíllo, C. Puyal, E., Elboj, C., Sanz, A., y Sanagustín, M. V. (2005). Comportamiento social de los estudiantes de educación no universitaria en la Comunidad Aragonesa. Estudio preliminar. http://www.educa.aragob.es/ryc/Convi. es/Descargas/INFORME\%20PRELIMINAR.pdf

Gómez Rivas, F. (2011). La normativa sobre convivencia escolar en España. Revista Amazónica, 4, Vol. VI, (1), pp. 149-185.

Govern de les Illes Balears. Assetjament escolar (Bullying), (2005). Palma: Conselleria de Presidència i Esports. Direcció General de l'oficina de defensa dels Dets del Menor

Gobierno Vasco (2004). Educación para la convivencia y la paz en los centros escolares de la Comunidad Autónoma del País Vasco. Vitoria-Gasteiz: Gobierno Vasco. Departamento de Empleo y Seguridad social-Departamento de Educación, Universidades e Investigación-Departamento de Cultura. http://www.hezkuntza. ejgv.euskadi.net/r43-2459/eu/contenidos/informacion/dig2/eu_5614/adjuntos/bakea/ ca_index.htm

Grasa, T., fuente, G., López, M. y Royo, F. (2006). Contigo. Convivencia entre iguales. Zaragoza: Departamento de Educación, Cultura y Deporte. 
Ley 29719 (2012). Ley que promueve la convivencia sin violencia en las instituciones educativas. Lima: Congreso de la República.

Mason, K. (2008). Cyberbullying: a preliminary assessment for shool personnel. En Psychology in the schools, Vol. 45 (4), 323-348.

Monjas, M.I. y Avilés, J.Ma (2006). Programa de sensibilización contra el maltrato entre iguales. Valladolid: Junta de Castilla y León.

Olweus, D. (1983). Low school achievement and aggressive behavior in adolescent boys. En D. Magnusson y V. Allen (Eds.), Human development. An interactional perspective (pp.353-365). New York: Academic Press.

Olweus, D. (1998). Conductas de acoso y amenaza entre escolares. Madrid: Morata.

Oñederra, J.A., Martínez, P., Tambo I., y Ubieta E. (2005b). El maltrato entre iguales, "Bullying" en Euskadi. Educación Secundaria. Gobierno Vasco. ISEI-IVEI. http:// www.isei-ivei.net

Ortega, R. (1992). Violence in Schools. Bully-victims Problems in Spain, Vth. European Conference on Developmental Psychology, (pp.27). Sevilla.

Ramírez, S. (2006). El maltrato entre escolares y otras conductas problema para la convivencia: Un estudio desde el contexto del grupo-clase. Tesis doctoral no publicada. Ceuta: Universidad de Granada.

Rigby, K. y Slee, P. T. (1999b). Suicidal ideation among adolescent school children, involvement in bully-victim problems, and perceived social support. Suicide and Life-Threatening Behavior, 29(2), 119-130.

Sáenz, T., Calvo, J., Fernández F., y Silván, A. (2005). El acoso escolar en los centros educativos de La Rioja. La Rioja: Informe inédito del Servicio de Inspección Técnica Educativa. Sector Rioja Baja-Logroño Este.

Salmivalli, C., Karhunen, J. y Lagerspetz, K.M.J. (1996). How do the victims resond to bullying?. Aggressive behaviour, 22, 99-109.

Smith, P.K. (1999) England and Wales. En P.K. Smith, Y. Morita, J. Junger-Tas, D. Olweus, R. Catalano y P. Slee (Eds.), The nature of school bullying, (pp. 68-90). London: Routlegde.

Smith, P. K., Pepler, D., Rigby, K. (2004). Bullying in schools. How successful can interventions be? Cambridge: Cambridge University Press.

Síndic de Greuges de la Comunidad Valenciana (2006). Estudio Epidemiológico del Bullying en la Comunidad Valenciana. Elaborado por E. Martín, F. Pérez, A. Marchesi, E. M. Pérez y N. Álvarez. Valencia: Sindic de Greuges.

Sullivan, K. (2001). The anti-bullying handbook. Singapore: Oxford University Press.

Uruñuela, P. y Avilés, J.M. (2012). El aprendizaje-servicio como estrategia global de mejora de la formación intregal del alumnado en la mejora de la convivencia. En J.L. Pais, I. Leal, A. Pereira, A. Torres, I. Direito y P. Vagos (Eds.), actas del $9^{\circ}$ Congreso de Psicología da Saude, pp. 957-962, Aveiro: Placebo. 
VISTA (2008): Violence in school taking action, (http://www.vista-europe.org/) (29 diciembre 2008)

VISTOP (2008). Violence in School Training Online Project, VISTOP (2006-2008). (http://www.vistop.org/) (14 enero 2007).

Zaitegi, N., Fernandez, I., Uruñuela, P., Avilés, J.M., Boqué, M.C., y Gómez, F. (2010). La educación en y para la convivencia positiva en España. En Revista iberoamericana sobre calidad, eficacia y cambio en la educación, (8), 2, pp- 93-132

Ziegler, S. y Rosenstein-Manner, M. (1991). Bullying at school: Toronto in an international context. Toronto board of education, 196R. 
